\title{
The Inertial Manifold for Class Kirchhoff-Type Equations with Strongly Damped Terms and Source Terms
}

\author{
Guoguang Lin, Xiangshuang Xia \\ Department of Mathematics, Yunnan University, Kunming, China \\ Email: gglin@ynu.edu.cn, 1527492605@qq.com
}

How to cite this paper: Lin, G.G. and Xia, X.S. (2018) The Inertial Manifold for Class Kirchhoff-Type Equations with Strongly Damped Terms and Source Terms. Applied Mathematics, 9, 730-737.

https://doi.org/10.4236/am.2018.96050

Received: April 11, 2018

Accepted: June 26, 2018

Published: June 29, 2018

Copyright (๑) 2018 by authors and Scientific Research Publishing Inc. This work is licensed under the Creative Commons Attribution International License (CC BY 4.0).

http://creativecommons.org/licenses/by/4.0/

\begin{abstract}
In this paper, we study the inertial manifolds for a class of the Kirchhoff-type equations with strongly damped terms and source terms. The inertial manifold is a finite dimensional invariant smooth manifold that contains the global attractor, attracting the solution orbits by the exponential rate. Under appropriate assumptions, we firstly exert the Hadamard's graph transformation method to structure a graph norm of a Lipschitz continuous function, and then we prove the existence of the inertial manifold by showing that the spectral gap condition is true.
\end{abstract}

\section{Keywords}

Inertial Manifold, Hadamard's Graph Transformation Method, Lipschitz Continuous, Spectral Gap Condition

\section{Introduction}

In this paper, we concerned the equation:

$$
\left\{\begin{array}{l}
u_{t t}-M\left(\|\nabla u\|^{2}+\left\|\nabla^{m} v\right\|^{2}\right) \Delta u-\beta \Delta u_{t}+g_{1}(u, v)=f_{1}(x), \\
v_{t t}+M\left(\|\nabla u\|^{2}+\left\|\nabla^{m} v\right\|^{2}\right)(-\Delta)^{m} v+\beta(-\Delta)^{m} v_{t}+g_{2}(u, v)=f_{2}(x), \\
u(x, 0)=u_{0}(x), \quad u_{t}(x, 0)=u_{1}(x), \quad x \in \Omega, \\
v(x, 0)=v_{0}(x), \quad v_{t}(x, 0)=v_{1}(x), \quad x \in \Omega, \\
\left.u\right|_{\partial \Omega}=0,\left.\quad v\right|_{\partial \Omega}=0,\left.\quad \frac{\partial_{i} v}{\partial \mu^{i}}\right|_{\partial \Omega}=0(i=1,2, \cdots, m-1),
\end{array}\right.
$$

where $\Omega$ is a bounded domain in $R^{n}$ with a smooth boundary $\partial \Omega, \beta>1$ is a 
constant and $f_{i}(x)(i=1,2)$ is a given source term. Moreover, $M\left(\|\nabla u\|^{2}+\left\|\nabla^{m} v\right\|^{2}\right)$ is a scalar function. Then the assumptions on $M$ and $g_{i}(u, v)$ will be specified later.

Nowadays, the study on the complexity of the space-time of high dimensional and infinite dimensional dynamical systems has gradually become the focus of nonlinear scientific research. In recent years, the inertial manifold has been found in the researches of the long time behavior of the solution and the attractor structure. The inertial manifold is a tool to describe the interaction between the low frequency components and the high frequency components [1]. When the flow has an inertial manifold, its high frequency description depends on the low frequency, and it contains attractors and exponentially attracts solution of the track, which realizes that the infinite dimensional dynamical system is reduced to a finite dimensional dynamical systems of the finite dimensional invariable Lipschitz manifold. Therefore, the inertial manifold is a powerful tool to study the long-time behavior of nonlinear dissipative systems and expose the real or seemingly chaotic structure of nonlinear dynamics.

In addition, the study of inertial manifold is of great significance. The central idea of the methods that people use to solve practical problems such as Galerkin method, Cellular automaton and Coupled map, are to discuss the infinite dimensional problem into a finite dimensional problem. So, the inertial manifold is of great significance to the development of nonlinear science.

In 1988, the concept of inertial manifold was first proposed in the study of infinite dimensional dynamical system by R. Temam, C. Foias and Sell G.R. [2]. They considered the equation as following:

$$
u_{t}+A u+B(u, u)+C(u)-f=0 .
$$

where $A u$ is a linear unbounded self-adjoint operator on $H$ with domain $D(A)$ dense in $H$.

In 2010, Guoguang Lin and Jingzhu Wu [3] studied the existence of the inertial manifold of Boussinesq equation:

$$
\left\{\begin{array}{l}
u_{t t}-\alpha \Delta u_{t}-\Delta u+u^{2 k+1}=F(x, y), \\
u(x, y, 0)=u_{0}(x, y), \\
u(x, y, t)=u(x+\pi, y, t)=u(x, y+\pi, t)=0, \quad(x, y) \in \Omega,
\end{array}\right.
$$

where $\Omega=(0, \pi)^{2} \in R^{2}, t>0, \alpha>2$.

In 2016, Ling Chen, Wei Wang and Guoguang Lin [4] established the exponential attractors and inertial manifolds of the higher-order Kirchhoff-type equation:

$$
u_{t t}+(-\Delta)^{m} u_{t}+\phi\left(\left\|\nabla^{m} u\right\|^{2}\right)(-\Delta)^{m} u+g(u)=f(x)
$$

There are many researches on inertial manifolds for nonlinear wave equations (see [5] [6]). Concerning the inertial manifold, many difficulties are solved. So we take advantage of Hadamard's graph transformation method in this paper.

The paper is arranged as follows. In Section 2, some assumptions, notations 
and lemmas are stated. In Section 3, the existence of the inertial manifold is established.

\section{Preliminaries}

For convenience, we first introduce the following notations:

$$
X=H_{0}^{2}(\Omega) \times H_{0}^{2 m}(\Omega) \times H_{0}^{1}(\Omega) \times H_{0}^{m}(\Omega), X_{0}=H_{0}^{1}(\Omega) \times H_{0}^{m}(\Omega),
$$

$c_{i}(i=1,2, \cdots)$ denotes different positive constants, $(\cdot, \cdot)$ and $\|\cdot\|$ are the inner product and norm of $L^{2}(\Omega),\|\cdot\|_{-m}$ is the norm of $H^{-m}(\Omega)$.

Next, we give some assumptions and definition needed in the proof of our results.

$$
\begin{gathered}
\left(A_{1}\right) g_{i}(u, v) \in C^{1}(\Omega), \quad(i=1,2) . \\
\left(A_{2}\right) \varepsilon \leq m_{0} \leq M(s) \leq m_{1} \leq \frac{(\beta-1) \mu_{k}}{4} .
\end{gathered}
$$

Definition 2.1. [7] Let $A: X \rightarrow X$ be an operator and assume that $F \in C_{b}(X, X)$ satisfies the Lipschitz condition

$$
\|F(U)-F(V)\|_{X} \leq l_{F}\|U-V\|_{X}, \quad U, V \in X .
$$

The operator $A$ is called satisfy the spectral gap condition relative to $F$, if the point spectrum of the operator $A$ can be divided into two parts $\sigma_{1}$ and $\sigma_{2}$, of which $\sigma_{1}$ is finite, and such that, if

$$
\Lambda_{1}=\sup \left\{\operatorname{Re} \lambda \mid \lambda \in \sigma_{1}\right\}, \Lambda_{2}=\inf \left\{\operatorname{Re} \lambda \mid \lambda \in \sigma_{2}\right\},
$$

and

$$
X_{i}=\operatorname{span}\left\{w_{j} \mid \lambda_{j} \in \sigma_{i}\right\}, \quad i=1,2 .
$$

Then

$$
\Lambda_{2}-\Lambda_{1}>4 l_{F},
$$

and the orthogonal decomposition $X=X_{1} \oplus X_{2}$ holds with continuous orthogonal projections $P_{1}: X \rightarrow X_{1}, P_{2}: X \rightarrow X_{2}$.

Lemma 2.1. [8] Let the eigenvalues $\mu_{j}^{ \pm}, j \geq 1$ be arranged in nondecreasing order. For all $\forall m \in N$, there exists $N \geq m$ such that $\mu_{N}^{-}$and $\mu_{N+1}^{-}$are consecutive.

\section{The Inertial Manifold}

Equation (1.1) is equivalent to the following one order evolution equation:

$$
U_{t}+A U=F(U) \text {, }
$$

where $U=(u, v, p, q)^{\mathrm{T}} \in X, p=u_{t}, q=v_{t}$,

$$
A=\left(\begin{array}{cccc}
0 & 0 & -I & 0 \\
0 & 0 & 0 & -I \\
-M(s) \Delta & 0 & -\beta \Delta & 0 \\
0 & M(s)(-\Delta)^{m} & 0 & \beta(-\Delta)^{m}
\end{array}\right), F(U)=\left(\begin{array}{c}
0 \\
0 \\
f_{1}(x)-g_{1}(u, v) \\
f_{2}(x)-g_{2}(u, v)
\end{array}\right)
$$




$$
D(A)=\left\{(u, v) \in H_{0}^{2}(\Omega) \times H_{0}^{2 m}(\Omega)\right\} \times H_{0}^{1}(\Omega) \times H_{0}^{m}(\Omega) .
$$

We consider the usual graph norm in $X$, as follows

$$
(U, V)_{X}=\left(M(s) \nabla u, \nabla \overline{u_{1}}\right)+\left(M(s) \nabla^{m} v, \nabla^{m} \overline{v_{1}}\right)+\left(\overline{p_{1}}, p\right)+\left(\overline{q_{1}}, q\right),
$$

where $U=(u, v, p, q)^{\mathrm{T}}, V=\left(u_{1}, v_{1}, p_{1}, q_{1}\right)^{\mathrm{T}} \in X, \overline{u_{1}}, \overline{v_{1}}, \overline{p_{1}}, \overline{q_{1}}$ respectively represent the conjugation of $u_{1}, v_{1}, p_{1}, q_{1}$. Evidently, the operator $A$ is monotone, for $U \in D(A)$, we obtain

$$
\begin{aligned}
(A U, U)_{X}= & (-M(s) \nabla p, \nabla \bar{u})+\left(-M(s) \nabla^{m} q, \nabla^{m} \bar{v}\right) \\
& +(\bar{p},-M(s) \Delta u-\beta \Delta p)+\left(\bar{q}, M(s)(-\Delta)^{m} v+\beta(-\Delta)^{m} q\right) \\
= & (-M(s) \nabla p, \nabla \bar{u})+(\nabla p, M(s) \nabla u)+\beta\|\nabla p\|^{2} \\
& +\left(-M(s) \nabla^{m} q, \nabla^{m} \bar{v}\right)+\left(\nabla^{m} \bar{q}, M(s) \nabla^{m} v\right)+\beta\left\|\nabla^{m} q\right\|^{2} \\
= & \beta\|\nabla p\|^{2}+\beta\left\|\nabla^{m} q\right\|^{2} \geq 0 .
\end{aligned}
$$

So, $(A U, U)_{X}$ is a nonnegative and real number.

In order to determine the eigenvalues of $A$, we consider the eigenvalues equation:

$$
A U=\lambda U, \quad U=(u, v, p, q)^{\mathrm{T}} \in X
$$

That is

$$
\left\{\begin{array}{l}
-p=\lambda u, \\
-q=\lambda v, \\
-M(s) \Delta u-\beta \Delta p=\lambda p, \\
M(s)(-\Delta)^{m} v+\beta(-\Delta)^{m} q=\lambda q .
\end{array}\right.
$$

Substitute (3.6), (3.7) into (3.8), (3.9), we obtain

$$
\left\{\begin{array}{l}
\lambda^{2} u-M(s) \Delta u+\beta \lambda \Delta u=0, \\
\lambda^{2} v+M(s)(-\Delta)^{m} v-\beta \lambda(-\Delta)^{m} v=0 .
\end{array}\right.
$$

Replacing $u, v$ with $u_{k}, v_{k}$, taking $u, v$ inner product with the Equations (3.9), (3.10), and adding them together, we have

$$
\lambda_{k}^{k}\left(\left\|u_{k}\right\|^{2}+\left\|v_{k}\right\|^{2}\right)+M(s)\left(\left\|\nabla u_{k}\right\|^{2}+\left\|\nabla^{m} v_{k}\right\|^{2}\right)-\lambda_{k} \beta\left(\left\|\nabla u_{k}\right\|^{2}+\left\|\nabla^{m} v_{k}\right\|^{2}\right)=0 .
$$

(3.11) is regard as a quadratic equation with one unknown about $\lambda_{k}$, so we get

$$
\lambda_{k}^{ \pm}=\frac{\beta \mu_{k} \pm \sqrt{\beta^{2} \mu_{k}^{2}-4 M(s) \mu_{k}}}{2},
$$

for $\forall k \geq 1$, we have

$$
\left\|u_{k}\right\|^{2}+\left\|v_{k}\right\|^{2}=1,\left\|\nabla u_{k}\right\|^{2}+\left\|\nabla^{m} v_{k}\right\|^{2}=\mu_{k},\left\|\nabla^{-1} u_{k}\right\|^{2}+\left\|\nabla^{-m} v_{k}\right\|^{2}=\frac{1}{\mu_{k}} .
$$

and $\mu_{k}$ is non-derogatory. If $\beta \geq \frac{4 M(s)}{\mu_{k}}+1$, because of $\beta>1$, then 
$\beta^{2} \geq \frac{4 M(s)}{\mu_{k}}$, we can get the eigenvalues of $A$ are all positive and real numbers.

The corresponding eigenfunction is as follows

$$
U_{k}^{ \pm}=\left(u_{k}, v_{k},-\lambda_{k}^{ \pm} u_{k},-\lambda_{k}^{ \pm} v_{k}\right) .
$$

Lemma 3.1. $g_{i}: X_{0} \rightarrow X_{0},(i=1,2)$ is uniformly bounded and globally Lipschitz continuous.

Proof. $\forall(u, v),\left(u_{1}, v_{1}\right) \in X_{0}$, by $\left(A_{1}\right)$, we have

$$
\begin{aligned}
& \left\|g_{i}(u, v)-g_{i}\left(u_{1}, v_{1}\right)\right\|_{X_{0}} \\
& \leq\left\|g_{i u}(\xi, v)\left(u-u_{1}\right)\right\|_{H_{0}^{1}(\Omega)}+\left\|g_{i v}(u, \eta)\left(v-v_{1}\right)\right\|_{H_{0}^{m}(\Omega)} \\
& \leq c_{i_{1}}\left\|u-u_{1}\right\|_{H_{0}^{1}(\Omega)}+c_{i_{2}}\left\|v-v_{1}\right\|_{H_{0}^{m}(\Omega)} \\
& \leq c_{i_{3}}\left(\left\|u-u_{1}\right\|_{H_{0}^{1}(\Omega)}+\left\|v-v_{1}\right\|_{H_{0}^{m}(\Omega)}\right),
\end{aligned}
$$

where

$$
c_{i_{3}}=\max \left\{c_{i_{1}}, c_{i_{2}}\right\}, \xi=u+\left(1-\theta_{1}\right) u_{1}, \eta=v+\left(1-\theta_{2}\right) v_{1} .
$$

Let $l_{i}=c_{i_{3}}$, then $l_{i}$ is Lipschitz coefficient of $g_{i}(u, v)$.

Theorem 3.1. $l_{i}$ is Lipschitz constant of $g_{i}(u, v)$, when $\beta \geq \frac{4 M(s)}{\mu_{k}}+1$, set $N_{1} \in N$, such that $N \geq N_{1}$, we obtain

$$
\left(\mu_{N+1}-\mu_{N}\right)\left(\frac{1}{2}-\frac{1}{2} \sqrt{(\beta-1) \mu_{1}-4 m_{1}}\right) \geq \frac{4 l}{\sqrt{(\beta-1) \mu_{1}-4 m_{1}}}+1,
$$

where $l=\max \left\{l_{1}, l_{2}\right\}$. By $\left(A_{2}\right)$ and Lemma 3.1, the operator $A$ satisfies the spectral gap condition of (2.4).

Proof. when $\beta \geq \frac{4 M(s)}{\mu_{k}}+1$, the eigenvalues of $A$ are all positive and real numbers, meanwhile $\left\{\lambda_{k}^{-}\right\}_{k \geq 1}$ and $\left\{\lambda_{k}^{+}\right\}_{k \geq 1}$ are increasing order.

Next, we divided the whole process of proof into four steps.

Step 1 By Lemma 2.1, since $\left\{\lambda_{k}^{ \pm}\right\}$is nondecreasing order, so there exists $N$, such that $\lambda_{N}^{-}$and $\lambda_{N+1}^{-}$are continuous. Then the eigenvalues of $A$ are separate as

$$
\sigma_{1}=\left\{\lambda_{j}^{-}, \lambda_{k}^{+} \mid \max \left\{\lambda_{j}^{-}, \lambda_{k}^{+}\right\} \leq \lambda_{N}^{-}\right\}, \sigma_{2}=\left\{\lambda_{j}^{-}, \lambda_{k}^{ \pm} \mid \lambda_{j}^{-} \leq \lambda_{N}^{-} \leq \min \left\{\lambda_{j}^{+}, \lambda_{k}^{ \pm}\right\}\right\} .
$$

Step 2 The corresponding $X$ is decomposed into

$$
X_{1}=\operatorname{span}\left\{U_{j}^{-}, U_{k}^{+} \mid \lambda_{j}^{-}, \lambda_{k}^{+} \in \sigma_{1}\right\}, X_{2}=\operatorname{span}\left\{U_{j}^{-}, U_{k}^{ \pm} \mid \lambda_{j}^{-}, \lambda_{k}^{ \pm} \in \sigma_{2}\right\} .
$$

We aim at madding two orthogonal subspaces of $X$ and verifying the spectral gap condition (2.4) is true when $\Lambda_{1}=\lambda_{N}^{-}, \Lambda_{2}=\lambda_{N+1}^{-}$. Therefore, we further decompose $X_{2}=X_{c} \oplus X_{R}$, where

$$
X_{c}=\operatorname{span}\left\{U_{j}^{-} \mid \lambda_{j}^{-} \leq \lambda_{N}^{-}<\lambda_{j}^{+}\right\}, X_{R}=\operatorname{span}\left\{U_{k}^{ \pm} \mid \lambda_{N}^{-}<\lambda_{k}^{ \pm}\right\} .
$$

Set $X_{N}=X_{1} \oplus X_{c}$, in order to verify the $X_{1}$ and $X_{2}$ are orthogonal, we 
need to introduce two functions $\Phi: X_{N} \rightarrow R, \Psi: X_{R} \rightarrow R$.

$$
\begin{aligned}
\Phi(U, V)= & \beta\left(\nabla u, \nabla \overline{u_{1}}\right)-4 M(s)\left(u, \overline{u_{1}}\right)+\left(\nabla^{-1} \overline{p_{1}}, \nabla u\right)+\left(\nabla^{-1} \bar{p}, \nabla u_{1}\right) \\
& +4\left(\nabla^{-1} p, \nabla^{-1} \overline{p_{1}}\right)+\beta\left(\nabla^{m} v, \nabla^{m} \overline{v_{1}}\right)-4 M(s)\left(v, \overline{v_{1}}\right) \\
& +\left(\nabla^{-m} \overline{q_{1}}, \nabla^{m} v\right)+\left(\nabla^{-m} \bar{q}, \nabla^{m} v_{1}\right)+4\left(\nabla^{-m} q, \nabla^{-m} \overline{q_{1}}\right), \\
\Psi(U, V)= & 2 \beta\left(\nabla u, \nabla \overline{u_{1}}\right)-\beta\left(\nabla^{-1} \overline{p_{1}}, \nabla u\right)-\beta\left(\nabla^{-1} \bar{p}, \nabla u_{1}\right) \\
& +\beta\left(\nabla^{-1} p, \nabla^{-1} \overline{p_{1}}\right)+2 \beta\left(\nabla^{m} v, \nabla^{m} \overline{v_{1}}\right)-\beta\left(\nabla^{-m} \overline{q_{1}}, \nabla^{m} v\right) \\
& -\beta\left(\nabla^{-m} \bar{q}, \nabla^{m} v_{1}\right)+\beta\left(\nabla^{-m} q, \nabla^{-m} \overline{q_{1}}\right),
\end{aligned}
$$

where $U, V \in X$ are defined before.

Let $U=(u, v, p, q) \in X_{N}$, by $\left(A_{2}\right)$, then

$$
\begin{aligned}
\Phi(U, U)= & \beta(\nabla u, \nabla \bar{u})-4 M(s)(u, \bar{u})+\left(\nabla^{-1} \bar{p}, \nabla u\right)+\left(\nabla^{-1} \bar{p}, \nabla u\right) \\
& +4\left(\nabla^{-1} p, \nabla^{-1} \bar{p}\right)+\beta\left(\nabla^{m} v, \nabla^{m} \bar{v}\right)-4 M(s)(v, \bar{v}) \\
& +\left(\nabla^{-m} \bar{q}, \nabla^{m} v\right)+\left(\nabla^{-m} \bar{q}, \nabla^{m} v\right)+4\left(\nabla^{-m} q, \nabla^{-m} \bar{q}\right) \\
\geq & (\beta-1)\left(\|\nabla u\|^{2}+\left\|\nabla^{m} v\right\|^{2}\right)-4 M(s)\left(\|u\|^{2}+\|v\|^{2}\right) \\
\geq & {\left[(\beta-1) \mu_{1}-4 m_{1}\right]\left(\|u\|^{2}+\|v\|^{2}\right) . }
\end{aligned}
$$

Since for $\forall k, m_{1} \leq \frac{(\beta-1) \mu_{k}}{4}$, therefore $\Phi(U, U) \geq 0$, for $\forall U \in X_{N}$, then $\Phi$ is positive definite.

Similarly, for $U \in X_{R}$, we have

$$
\begin{aligned}
\Psi(U, U)= & 2 \beta(\nabla u, \nabla \bar{u})-\beta\left(\nabla^{-1} \bar{p}, \nabla u\right)-\beta\left(\nabla^{-1} \bar{p}, \nabla u\right) \\
& +\beta\left(\nabla^{-1} p, \nabla^{-1} \bar{p}\right)+2 \beta\left(\nabla^{m} v, \nabla^{m} \bar{v}\right)-\beta\left(\nabla^{-m} \bar{q}, \nabla^{m} v\right) \\
& -\beta\left(\nabla^{-m} \bar{q}, \nabla^{m} v\right)+\beta\left(\nabla^{-m} q, \nabla^{-m} \bar{q}\right) \\
\geq & \beta\left(\|\nabla u\|^{2}+\left\|\nabla^{m} v\right\|^{2}\right) \geq \beta \mu_{1}\left(\|u\|^{2}+\|v\|^{2}\right) .
\end{aligned}
$$

So, for $\forall U \in X_{R}, \Psi(U, U) \geq 0$, the $\Psi$ is also positive definite.

Next, we need to define a scale product in $X$

$$
\langle\langle U, V\rangle\rangle_{X}=\Phi\left(P_{N} U, P_{N} V\right)+\Psi\left(P_{R} U, P_{R} V\right),
$$

where $P_{N}$ and $P_{R}$ are projection: $X \rightarrow X_{N}, X \rightarrow X_{R}$ respectively, for convenience, we rewrite (3.24) as follows

$$
\langle\langle U, V\rangle\rangle_{X}=\Phi(U, V)+\Psi(U, V) .
$$

We will proof that two subspaces $X_{1}$ and $X_{2}$ in (3.18) are orthogonal. In fact, we only need to show $X_{N}$ and $X_{C}$ are orthogonal, that is

$$
\left\langle\left\langle U_{j}^{-}, U_{j}^{+}\right\rangle\right\rangle_{X}=0 \quad\left(\forall U_{j}^{-} \in X_{N}, U_{j}^{+} \in X_{c}\right) .
$$

By (3.20), (3.25), we have 


$$
\begin{aligned}
\left\langle\left\langle U_{j}^{-}, U_{j}^{+}\right\rangle\right\rangle_{X}= & \Phi\left(U_{j}^{-}, U_{j}^{+}\right) \\
= & \beta\left(\nabla u_{j}, \nabla \overline{u_{j}}\right)-4 M(s)\left(u_{j}, \overline{u_{j}}\right)-\lambda_{j}^{+}\left(\nabla^{-1} \overline{u_{j}}, \nabla u_{j}\right)-\lambda_{j}^{-}\left(\nabla^{-1} \overline{u_{j}}, \nabla u_{j}\right) \\
& +4 \lambda_{j}^{-} \lambda_{j}^{+}\left(\nabla^{-1} u_{j}, \nabla^{-1} \overline{u_{j}}\right)+\beta\left(\nabla^{m} v_{j}, \nabla^{m} \overline{v_{j}}\right)-4 M(s)\left(v_{j}, \overline{v_{j}}\right) \\
& -\lambda_{j}^{+}\left(\nabla^{-m} \overline{v_{j}}, \nabla^{m} v_{j}\right)-\lambda_{j}^{-}\left(\nabla^{-m} \overline{v_{j}}, \nabla^{m} v_{j}\right)+4 \lambda_{j}^{-} \lambda_{j}^{+}\left(\nabla^{-m} v_{j}, \nabla^{-m} \overline{v_{j}}\right) \\
= & \beta\left(\left\|\nabla u_{j}\right\|^{2}+\left\|\nabla^{m} v_{j}\right\|^{2}\right)-M(s)\left(\left\|u_{j}\right\|^{2}+\left\|v_{j}\right\|^{2}\right) \\
& -\left(\lambda_{j}^{+}+\lambda_{j}^{-}\right)\left(\left\|u_{j}\right\|^{2}+\left\|v_{j}\right\|^{2}\right)+4 \lambda_{j}^{-} \lambda_{j}^{+}\left(\left\|u_{j}\right\|_{-1}^{2}+\left\|v_{j}\right\|_{-m}^{2}\right) .
\end{aligned}
$$

By (3.12), we can get $\lambda_{j}^{+}+\lambda_{j}^{-}=\beta \mu_{j}, \lambda_{j}^{-} \lambda_{j}^{+}=M(s) \mu_{j}$, therefore

$$
\left\langle\left\langle U_{j}^{-}, U_{j}^{+}\right\rangle\right\rangle_{X}=\Phi\left(U_{j}^{-}, U_{j}^{+}\right)=0 .
$$

Step 3 Further, we estimate the Lipschitz constant $l_{F}$ of $F(U)$ (3.2). According to Lemma 3.1, $g_{i}: X_{3} \rightarrow X_{3}$ is Lipschitz continuous with Lipschitz constant $l_{i}$. Let $P_{i}: X \rightarrow X_{i}(i=1,2)$ is orthogonal projection. From (3.22), (3.23) and (3.24), we have

$$
\begin{aligned}
\|U\|_{X}^{2} & =\Phi\left(P_{1} U, P_{1} U\right)+\Psi\left(P_{2} U, P_{2} U\right) \\
& \geq\left[(\beta-1) \mu_{1}-4 m_{1}\right]\left(\left\|P_{1} u\right\|^{2}+\left\|P_{1} v\right\|^{2}\right)+\beta \mu_{1}\left(\left\|P_{2} u\right\|^{2}+\left\|P_{2} v\right\|^{2}\right) \\
& \geq\left[(\beta-1) \mu_{1}-4 m_{1}\right]\left(\left\|P_{1} u\right\|^{2}+\left\|P_{2} u\right\|^{2}+\left\|P_{1} v\right\|^{2}+\left\|P_{2} v\right\|^{2}\right) \\
& \geq\left[(\beta-1) \mu_{1}-4 m_{1}\right]\left(\|u\|^{2}+\|v\|^{2}\right) .
\end{aligned}
$$

Given $U=(u, v, p, q)^{\mathrm{T}}, V=\left(u_{1}, v_{1}, p_{1}, q_{1}\right)^{\mathrm{T}} \in X$, we have

$$
\begin{aligned}
\|F(U)-F(V)\|_{X} & =\left\|g_{1}(u, v)-g_{1}\left(u_{1}, v_{1}\right)\right\|_{X_{3}}+\left\|g_{2}(u, v)-g_{2}\left(u_{1}, v_{1}\right)\right\|_{X_{3}} \\
& \leq l_{1}\left(\left\|u-u_{1}\right\|+\left\|v-v_{1}\right\|\right)+l_{2}\left(\left\|u-u_{1}\right\|+\left\|v-v_{1}\right\|\right) \\
& \leq l\left(\left\|u-u_{1}\right\|+\left\|v-v_{1}\right\|\right) \\
& \leq \frac{l}{\sqrt{(\beta-1)-4 m_{1}}}\|U-V\|_{X},
\end{aligned}
$$

where $l=\max \left\{l_{1}, l_{2}\right\}$.

So, we obtain

$$
l_{F} \leq \frac{l}{\sqrt{(\beta-1)-4 m_{1}}} .
$$

Step 4 Now, we will show the spectral gap condition (2.4) holds.

Since $\Lambda_{1}=\lambda_{N}^{-}, \Lambda_{2}=\lambda_{N+1}^{-}$, then

$$
\Lambda_{2}-\Lambda_{1}=\lambda_{N+1}^{-}-\lambda_{N}^{-}=\frac{1}{2}\left(\mu_{N+1}-\mu_{N}\right)+\frac{1}{2} \sqrt{Q(N)}-\sqrt{Q(N+1)},
$$

where $Q(N)=\beta^{2} \mu_{N}^{2}-4 M(s) \mu_{N}$.

Let $N_{1}>0$, for $\forall N \geq N_{1}$, then

$Q_{1}(N)=1-\sqrt{\frac{\beta^{2}}{(\beta-1) \mu_{1}-4 m_{1}}-\frac{4 m_{1}}{\mu_{N}\left[(\beta-1) \mu_{1}-4 m_{1}\right]}}$, we can obtain 


$$
\begin{aligned}
& \sqrt{Q(N)}-\sqrt{Q(N+1)}+\sqrt{(\beta-1)-4 m_{1}}\left(\mu_{N+1}-\mu_{N}\right) \\
& =\sqrt{(\beta-1) \mu_{1}-4 m_{1}}\left(\mu_{N+1} Q_{1}(N+1)-\mu_{N} Q_{1}(N)\right)
\end{aligned}
$$

From $\left(A_{2}\right)$, we can easily obtain

$$
\lim _{N \rightarrow \infty}\left(\sqrt{Q(N)}-\sqrt{Q(N+1)}+\sqrt{(\beta-1) \mu_{1}-4 m_{1}}\left(\mu_{N+1}-\mu_{N}\right)\right)=0 .
$$

Then, according to (3.16), (3.31), (3.32) and (3.34), we have

$$
\begin{aligned}
\Lambda_{2}-\Lambda_{1} & >\left(\mu_{N+1}-\mu_{N}\right)\left(\frac{1}{2}-\frac{1}{2} \sqrt{(\beta-1) \mu_{1}-4 m_{1}}\right)-1 \\
& \geq \frac{4 l}{\sqrt{(\beta-1) \mu_{1}-4 m_{1}}} \geq 4 l_{F} .
\end{aligned}
$$

Therefore, Theorem 3.1 is true.

Theorem 3.2. Under the condition of Theorem 3.1, the problem (1.1)-(1.5) exist an inertial manifold $\mu$ in $X$,

$$
\mu=\operatorname{graph}(m):=\left\{\zeta+m(\zeta): \zeta \in X_{1}\right\},
$$

where $X_{1}, X_{2}$ defined in (3.18) and $m: X_{1} \rightarrow X_{2}$ is a Lipschitz continuous function.

\section{Acknowledgements}

The authors would like to thank for the anonymous referees for their valuable comments and suggestions sincerely. These contributions increase the value of the paper.

\section{References}

[1] Chen, F.S., Cai, W.K. and Cheng, W.S. (1995) The Inertial Manifold Is Applied to Scientific Calculation. Journal of Shanghai Electric Power Institute, 11, 15-24.

[2] Foias, C., Sell, G.R. and Temam, R. (1988) Inertial Manifold for Nonlinear Evolutionary Equations. Journal of Differential Equations, 73, 309-353. https://doi.org/10.1016/0022-0396(88)90110-6

[3] Wu, J.Z. and Lin, G.G. (2010) The Inertial Manifold of The Two-Dimensional Strong Damping Boussinesq Equation. Journal of Yunnan University, 32, 119-124.

[4] Chen, L., Wang, W. and Lin, G.G. (2016) Exponential Attractors and Inertial Manifolds for the Higher-Order Nonlinear Kirchhoff-Type Equation. International Journal of Modern Communication Technologies \& Research, 4, 6-12.

[5] Xu, G.G., Wang, L.B. and Lin, G.G. (2014) Inertial Manifolds for a Class of the Retarded Nonlinear Wave Equations. Mathematica Applicata, 27, 887-891.

[6] Lou, R.J., Lv, P.H. and Lin, G.G. (2016) Exponential Attractors and Inertial Manifolds for a Class of Generalized Nonlinear Kirchhoff-Sine-Gordon Equation. Journal of Advances in Mathenatics, 12, 6362-6374.

[7] Robinson, J.C. (2001) Infinite Dimensional Dynamical Systems. Cambridge University Press, London. https://doi.org/10.1007/978-94-010-0732-0

[8] Zheng, S.M. and Milani, A. (2004) Exponential Attractors and Inertial Manifolds for Singular Perturbations of the Cahn-Hilliard Equations. Nonlinear Analysis. Theory, Methods \& Applications, 57, 843-877.

https://doi.org/10.1016/j.na.2004.03.023 